\title{
Dynamics of attentional control
}

\author{
Hermannn J. Müller · Thomas Geyer
}

Published online: 9 December 2008

(C) Springer-Verlag 2008

Humans are continuously encountering a myriad of events at any given time in their environment, providing a huge load on the brain in processing the various inputs, linking (some of) them to representations stored in memory, and organizing appropriate responses (to some of them). However, while the brain has an impressive capability of parallel processing, its capacity is strongly limited. Thus, humans cannot consciously represent all the information available at any one time and they cannot initiate, at the same time, more than a few different actions. In order to overcome these limitations, humans have to focus on some limited subset of the whole perceptual array. The neurocognitive mechanisms that mediate this selection are referred to as 'attention'.

In the past decades, attention has become one of the most investigated areas in perception, cognition, and action. The purpose of this Psychological Research Special Issue is to present a selective overview of some of the main lines of current research on attention, with a focus on perceptual selectivity and visual selection in particular. Specifically, this Special Issue presents a set of papers concerned with four issues: (1) the relationship between overt and covert attention; (2) attention and memory; (3) control of attention based on stimulus properties and/or observers' goals; and (4) attention and object coding.

\footnotetext{
H. J. Müller $(\bowtie) \cdot$ T. Geyer $(\square)$

Department of Psychology, University of Munich, Leopoldstrasse 13, 80802 Munich, Germany

e-mail: hmueller@psy.lmu.de

T. Geyer

e-mail: geyer@lmu.de

H. J. Müller

School of Psychology, Birkbeck College,

University of London, London, UK
}

The papers are based on the presentations given by some 20 leading experts on attention, from a variety of disciplines-including experimental and neuropsychology, electro- and neurophysiology, functional imaging, and computational modeling — at the "Dynamics of Attentional Control" symposium held at Holzhausen/Ammersee, near Munich, Germany, June 15-17, 2007. The aim of this meeting was to foster a dialog amongst these experts, in order to contribute to identifying theoretically important joint issues and discuss ways of how these issues can be resolved by using convergent, integrated methodologies. The meeting was supported by the German Research Foundation ('Deutsche Forschungsgemeinschaft-DFG') as part of the DFG research group 'FOR 480' (University of Munich, Germany; coordinator: H. J. Müller).

\section{Structure of the special issue}

Covert and overt attention

It is widely accepted to draw a distinction between 'overt' and 'covert' visual attention. Overt attention is the act of directing the head and the eyes (the foveae) towards particular locations or objects in the scene. Covert attention is the act of mentally focusing on one of several possible locations/objects (in the absence of overt orienting movements). The current view is that covert visual attention is a mechanism for rapid scanning of the visual field for interesting objects/locations, with shifts in covert attention being linked to the eye movement circuitry setting up saccades to those locations/objects at a later point in time. In support of this view, the paper by J. Findlay shows that covert visual attention and saccade programming are closely related, by taking into account four types of control signals 
for the (covert) allocation of spatial attention: exogenous stimulus-driven signals and endogenous location, object-, and feature-based signals that can all influence the saccade generation process.

The subsequent paper by R. Kliegl, M. Rolfs, J. Laubrock, and $R$. Engbert explores whether microsaccades are indexing the allocation of (covert) spatial attention-an issue about which there has been a considerable controversy in the literature. The study of Kliegl et al. contributes to this debate by demonstrating, in four variations of Posner's spatial-cueing paradigm (auditory cue-auditory target; auditory cue-visual target; visual cue-auditory target; visual cue-visual target), critical links between the location, number, and latency of microsaccades and the location of the (auditory) target relative to the cue. Kliegl et al. conclude that microsaccades can be considered as a marker of spatial attention, with a 'weak' version of this hypothesis providing the most parsimonious account of the available data.

The final paper in this section by R. M. Müri, D. Cazzoli, T. Nyffeler, and T. Pflugshaupt re-examines the oculomotor scanning behavior of patients with visual hemi-neglect. Due to temporo-parietal damage in the right brain hemisphere, such patients are typically unable to detect or respond to stimuli in the contralesional, left hemi-field. Analyzing eye movement patterns in 15 neglect patients presented with naturalistic color photographs of everyday scenes, Müri et al. provide important insights in how neglect patients explore, or fail to explore, their environment. In particular, they find the spatial distribution of fixations in neglect patients to be influenced not only by horizontal direction (i.e., fewer fixations in the left relative to the right hemifield), but also by vertical direction (i.e., even fewer in the bottom-left relative to the top-left hemifield).

\section{Attention and memory}

The present Special Issue places special emphasis on the relations between attention and memory, in particular, memory-based guidance of attention in visual search. In such tasks, observers usually have to detect a target item which is presented in an array of distractor items. Typically, performance is found to be dependent on specific properties of the search display (e.g., the number of display items) and whether observers can make use of a constant target template to guide their search. Over the past 1015 years, however, growing evidence has emerged that, besides bottom-up (saliency-driven) and top-down (goalmediated) guidance, memory mechanisms can also exert strong influences on the control of attention. In particular, (implicit) visual short-term memory has been shown to either speed up or slow search across consecutive trials ('priming') or sequences of trials ('perceptual learning'), modulating the processing of item locations, dimensions, features, and target-distractor arrangements, respectively.

The paper by A. Kristjansson focuses on the facilitatory effect associated with the repetition (relative to a change) of stimulus features across consecutive trials. More precisely, he explores whether and how featural priming in visual pop-out search (a special version of the visual search paradigm where the target is defined by a unique feature-such as a single red bar amongst multiple green bars) is influenced by task relevance of the target's defining dimension (color, motion direction). Kristjánsson demonstrates that featural priming is overall larger for task-relevant relative to irrelevant target attributes, with the beneficial effect resulting from the repetition of the task-relevant features being independent from the repetition of the irrelevant features.

In a similar vein, by examining positional and featural priming across longer time spans (i.e., the preceding five trials relative to the current trial), the paper by Geyer and Müller shows that priming of positions and features are independent phenomena. A second major outcome of their paper is that priming can be modulated intentionally: the effects of position or feature repetition were temporally extended when target positions or features were predictable, rather than unpredictable (i.e., at chance level), across trials.

Target location repetition effects are also at the heart of the paper by K. Finke, L. Bucher, G. Kerkhoff, I. Keller, F. von Rosen, T. Geyer, H. J. Müller, and P. Bublak. Their paper examines the facilitatory and inhibitory effects associated with the presentation of the target at a previous target location (facilitation) and, respectively, distractor location (inhibition) in a group of 14 patients with visual hemineglect. While facilitatory priming was preserved in these patients, inhibitory priming was strongly reduced (relative to an age-, education-, and IQ-matched control group). To explain this dissociation, Finke et al. propose that facilitatory and inhibitory locational priming rely on distinct frames of reference, with space-based representations underlying facilitatory priming and object-based representations inhibitory priming.

The next two papers in this section are concerned with the dimension repetition effect in visual search. This effect refers to the observation that, in cross-dimension search (where the target dimension is variable across trials), discerning the presence of a target is expedited when the current target is defined in the same dimension as the preceding target (no matter whether successive targets are featurally the same or different), compared to when it is defined in a different dimension. The paper by $J$ Krummenacher, H. J. Müller, M. Zehetleitner, and T. Geyer extends the investigation of this effect to compound tasks (where 
the detection-relevant attribute of the target-e.g., its unique color-is independent of the response-relevant attribute-e.g., the target's form). It is found that dimension repetition effects, although reliable, are reduced in compound relative to detection tasks (where observers simply have to decide on the presence vs. absence of the target). Closer inspection of the effects associated with repetitions/ changes of target location reveals that (1) target location repetition effects are overall larger in the compound task and that (2) reliable dimension repetition effects are evident only within a narrow region around the previous target location. To explain this pattern, Krummenacher et al. suggest that when the tasks makes high demands on focal attentional processing, (narrow) space-based weighting processes modulate the manifestation of (spatially parallel) dimension weighting processes.

The main questions addressed in the paper by $R$. Weidner and H. J. Müller are whether dimension repetition effects extend to conjunctive search tasks (where the target is defined by a combination of features-such as size and color) and, if so, whether there are beneficial effects associated with the presentation of redundantly defined conjunctive targets (i.e., multi-dimensional signal coactivation). To examine this, one (primary) target dimension (size) was held constant, while another (secondary) target dimension (color, motion) was made variable across trials (e.g., the singleton target could be large and red and presented amongst small and red together with large and green distractors). Weidner and Müller found dimension repetition effects (as revealed by the repetition relative to a change of the secondary target dimension) to be larger in this task, relative to simple feature pop-out tasks, and signal co-activation was observed. The results are explained in terms of dimension weighting, specifically: the larger RT gains associated with a repetition of the secondary target dimension in singleton conjunction search are attributed to a reduction in processing weight being available for the secondary dimension, due to a large amount of weight being bound by the (requirement for filtering in the) primary dimension.

The paper by A. A. Manginelli and S. Pollmann is concerned with long-term visual memory for target-distractor arrangement ('contextual cueing'). Previous research has shown that target discrimination can be expedited when the target appears within identically composed ('consistent') relative to newly composed ('inconsistent) target-distractor arrangements. While the basic observation is not in question, there has been a debate about the mechanisms that may underlie the contextual cueing effect: 'low-level' repetition priming (i.e., habituation to identical distractor locations) versus associative memory for target-distractor relations (arrangement). Manginelli and Pollmann examine these two alternative accounts by changing the position of the target (but keeping the distractors at the very same loca- tions) after observers have learned consistent target-distractor relations. The results from both RTs and eye movement measures are in support of the associative-learning account.

The final paper in this section by R. Perron, C. Lefebvre, N. Robitaille, B. Brisson, F. Gosselin, M. Arguin, and $P$. Jolicoeur examines two components of the event-related potential (ERP) - the N2pc (a negativity around 200-300 ms following stimulus onset at contralateral posterior electrodes) and the SPCN (a posterior contralateral negativity following the N2pc). Specifically, using a same-different task (observers had to decide whether two successively presented color arrays were the same or different), Perron et al. meant to assess whether the two ERP components- $\mathrm{N} 2 \mathrm{pc}$ and $\mathrm{SPCN}-$ are indexing different cognitive functions involved in the task: attention and memory, respectively. The results show a complex pattern of similarities and differences between the N2pc and SPCN, consistent with the view that the N2pc reflects mechanisms of attentional selection, whereas the SPCN reflects maintenance in visual short-term memory.

Top-down control of attention

While it is generally accepted that stimulus-driven and goal-mediated mechanisms of attention can influence target detection in visual search, there has been a great deal of interest recently in whether and how these mechanisms interact with each other in singleton feature search. On the one hand, salient feature singletons were claimed to capture attention automatically; on the other, it was proposed that bottom-up attentional capture by salient feature singletons can be modulated intentionally. The first two papers in this Section re-examine whether observers are able to ignore salient feature singletons.

In the study of A. Schubö, observers were instructed to identify a target item differing from distractor items in one of two dimensions (color, form) and respond according to the orientation of a line presented within the target item (i.e., compound task). On some trials, a distractor defined within the non-target dimension was presented in addition to the target. RTs as well ERP modulations $(\mathrm{N} 2 \mathrm{pc}, \mathrm{N} 1$, and N2-the latter two negativities are usually taken to reflect the operation of attention around 80-120 and, respectively, 150-300 ms post stimulus onset) were investigated dependent on the definition of an item as the to-be-processed target or the to-be-ignored distractor. The results showed that $\mathrm{N} 1$ and $\mathrm{N} 2$ components were generally larger on target only, compared to target plus singleton distractor, trials. RTs and N2pc results, by contrast, indicated a distractorinduced attention modulation that was only observed with form targets and color distractors, but not with color targets and form distractors. To explain this pattern, Schubö proposes that early ERP modulations $(\mathrm{N} 1, \mathrm{~N} 2)$ reflect 
saliency-independent feature contrast computations and late modulations (N2pc) saliency-dependent allocation of attention.

The paper by A. von Mühlenen and M. Conci also explores whether color singletons capture visual attention in a stimulus-driven manner. The search displays comprised of three or six letter elements (black color) presented on top of three or six disk elements (red or green color), with one disk being always different in color. The predefined target letter could appear either at the location of the singleton disk or at a non-singleton disk location. In line with previous reports, it is found that the presence of color singletons leads to capture of attention (as indexed by a steeper slope of the function relating RTs to display size on distractor, compared to target, singleton trials). However, further investigation of the capture effect revealed that the effect is critically dependent on the singleton and, in particular, the non-singleton items switching color between trials (e.g., between red and green), as compared to the singleton and non-singleton colors remaining constant across trials. This leads von Mühlenen and M. Conci to propose that the ability of (color) singletons to capture attention is modulated by whether observers are able to establish an appropriate (color-based) top-down set.

The issue of (endogenous) control of attention is also at the heart of the paper by J. Miller, D. Beutinger, and $R$. Ulrich, which examines the redundant-signal effect (RSE), that is, the speeding-up of RTs in the presence of dual relative to single targets. Miller et al. presented their observers with central arrow cues (pointing, e.g., to the left or the right), followed by one or two target squares on two imaginary rows (top or bottom row), with three possible positions of the square(s) within the row (left, right, or redundantly on both sides). Based on theoretical considerations concerning the top-down effects of spatial attention on stimulus processing, the RSE was hypothesized to be larger for unexpected relative to expected locations. However, the results showed this not to be case (i.e., RT redundancy gains with dual relative to single targets were statistically as large for cued as for uncued locations). From this, Miller et al. conclude that the RSE occurs at a stage after visual selection, where responses have already been translated into motor commands (i.e., response execution).

\section{Attention and object coding}

The first paper in the final section of this Special Issue by G. M. Jackson, R. Swainson, D. Mort, M. Husain, and S. R. Jackson examines attentional selection in a patient with simultanagnosia (following bilateral occipito-parietal damage). Typically, simultanagnosic patients are unable to perceive multiple items in a visual display, while their ability to perceive letters presented in isolation remains intact. Based on a number of experiments (ranging from visual search to letter identification tasks), Jackson et al. propose an account of simultanagnosia along the lines of the 'biased-competition hypothesis'. According to this hypothesis, due to the limited processing capacity of the visual system, multiple visual objects compete for neural representation. In order to select relevant information, irrelevant information must be suppressed, a process involving biasing the competition for selection against the irrelevant, and in favor of the relevant, information. When processing resources are significantly reduced, as in simultanagnosia, this competition is assumed to be particularly fierce, leading in extreme to the perception of single objects only.

The two following papers are concerned with the relations between spatial attention and figural coding processes. H. J. Müller and R. O'Grady re-investigated selective attention in the 'ring-cueing' paradigm of Egly and Homa (1984). Observers were cued to attend to one of three concentric rings of radius $1^{\circ}, 2^{\circ}$, or $3^{\circ}$, and their signal detection accuracy for cued and uncued rings was measured. In some conditions, the cue was a central color stimulus indicating a like-colored ring; in other conditions, the rings were monochrome and (the radius of) one of them was symbolically indicated a central size cue. With color cues to like-colored rings, the availability of secondary-depth information (provided by the display layout) had no influence on the ring-cueing effects. However, with monochrome rings and central size cues, the ring-cueing effects were significantly reduced when the depth information was disrupted. These results suggest that selection of a ring was object-based, operating on a spatial representation of the cued ring made salient by color or depth-based segmentation mechanisms.

The issue addressed in the paper of F. Bauer, M. Usher, and H. J. Müller is whether endogenous spatial attention influences (subliminal) entrainment of figural binding mechanisms. To examine this, Bauer et al. employed a spatial-cueing paradigm in which they examined the effects of valid and invalid spatial cues on temporal object priming (TOP). TOP refers to the observation that target detection can be expedited when the target figure is preceded, at its location, by figurally ambiguous 'premask' elements that are recycled synchronously (globally at $40 \mathrm{~Hz}$ ), rather than asynchronously. The results showed that TOP was larger for invalidly relative to validly cued locations, and that the TOP effect was figural, rather than spatial, in nature. From this, Bauer et al. argue that target processing is expedited not by attracting spatial attention to the primed location, but by the prime expediting (figure-specific) target encoding, as a result of which the target position gains a processing and selection advantage relative to non-primed locations. 